\title{
INTEGRATED CONSTRUCTION PROJECT MANAGEMENT SYSTEM BASED ON IFC AND ISO9001:2000
}

\author{
K. Umut Gökçe \& Raimar J. Scherer \\ Institute for Construction Informatics, Technology University Dresden, GERMANY, \\ \{Raimar.J.Scherer,Umut Gokce\}@cib.bau.tu-dresden.de \\ H. Attila Dikbaş \\ Project Management Center, Istanbul Technical University,TURKEY, \\ dikbas@itu.edu.tr
}

\begin{abstract}
We present a conceptual framework enabling to manage broad set of activities supported by multi-module software application for construction project management. The proposed structure is based on a common building information model (the industry standard IFC Model - ISO PAS 16739), the ISO 9001:2000 Quality Management System, for high-level process specification, and an integrated software infrastructure encompassing the product and process information exchange among the CAD-ERP and Scheduling Systems that support IFC. In this context, we developed Construction Management Phases for Software Interoperability, Organizational and IT Management Processes with using of ARIS methodology in order to implement IFC views. Based on this, we outline a web-based environment enabling to plug in all component tools via a common client, providing a coherent GUI.
\end{abstract}

\section{INTRODUCTION}

Today the actors in the construction environment use a great number of different media in an unstructured way which lowers the efficiency of the process and limits access to required information. Therefore, there is a need to describe the building, its parts and the management processes with multiple qualities. To make this description requires common concepts for the definition of standardized building objects, management aspects and relations between them.

In the last years, the aim to achieve higher degree of integration in the design and construction process has led to the development of a number of product and process models and integration architectures. Following early suggestions such as the IRMA model (Luiten et al. 1993), many national and international projects as ATLAS, ToCEE, ISTforCE, ICSS and ARKOS etc. have developed models of increasing complexity, targeting various aspects of interoperability. Supported through these efforts, the industry-driven IFC (industry foundation classes) model was born in the 90 s. This model is continuously improving and maturing towards a true standard for cooperative model-based working processes in AEC/FM (Liebich et al. 2006).

Gökçc, K.U., Schcrer, R.J., Dikbas, H.A., 2007, in IFIP International Federation for Information Processing, Volume 243, Establishing the Foundation of Collaborative Networks; eds. Camarinha-Matos, L., Afsarmanesh, H., Novais, P., Analide, C.; (Boston: Springer), pp. 513-520. 
However, in spite of all achievements for managing the process, product, documentation and communication, the organizational and information infrastructure in the AEC sector is still highly fragmented.

Currently in all industry countries there exist solutions integrating CAD, ERP scheduling and management tools. But, they largely lack generality in terms of data and process interoperability. ICT-supported construction project management (CPM) processes are mainly defined in terms of the used applications, and not on the basis of generalised industry requirements; similarly, integration of product and process information is based on the specific internal data models, and not on generally applicable and hence standardised data structures. All this significantly decreases flexibility, information exchange between the component systems, and last but not least, inter-enterprise cooperation and knowledge transfer.

Based on the determined requirements and acquiescence, to maintain an integrated structure to enable interoperable use of standard data in a generic CPM model in this research, we proposed an IT environment which is based on a formal process methodology, standardized product and process model (IFC), and overall architecture integrating technical (design) work, construction process planning and project management in a web-based structure, enabling to plug in all component tools via a common client providing a coherent GUI.

\section{OBJECTIVES}

To achieve interoperability in the area of CPM it is necessary to describe the building products, their parts and the related processes with multiple inter-related features. This requires taking into consideration (1) the economic and technical aspects, which can affect the products and processes during their lifecycle, and (2) the different involved discipline domains.

In this context, based on the experience gained from studying state-of-the art systems and best practice examples, the operational objectives for the development of an efficient Integrated CPM solution can be defined as follows:

1. Generalize and formally describe CPM processes so that interoperability over a broad spectrum of applications is facilitated,

2. Establish a common information model for CPM, based on the data schemas of the IFC standard (ISO/PAS 16739), thereby providing for the needed integration of product, process, cost and management data,

3. Provide interoperability methods to integrate legacy systems,

4. Develop a CPM assistance tool to interactively prove and ensure contextrelevant data completeness.

\section{APPROACH}

The specific requirements, the highly distributed nature of the construction industry, and the independently used systems for management processes provide the rationale for setting up the basic principles of the proposed systems.

In this research, a feasible methodology for interoperability was developed according to: (1) The IFC model of the IAI for a hierarchically structured product model, (2) The ISO Quality Management System (ISO 9001:2000) for the existing 
real-world process specification for managing CPM requirements of outcome and (3) Web-based integrated methods for encompassing the product and process information exchange within the CAD and CPM systems that support IFCs.

In order to constitute an integrated CPM Model, the Construction Management Phases for Software Interoperability (CMPSI) was formalized with using of IDEF0 modeling methodology according to implied requirements.

ISO9001:2000 Quality Management System Procedures were established subsequently, to support organizational management structure and to establish a control mechanism.

In order to narrow the scope and to better define the CPM aspects, the Bidding Preparation Phase (BPP) of CMPSI was chosen and the overall BPP processes were formalized in two interrelated subsystems using ARIS methodology (1996): (1) Organizational Management Process (OMP) and (2) IT Management processes (ITMP). To provide completeness between these interrelated systems a mapping structure between CMPSI, OMP and ITMP was also obtained.

The OMP provides the core process structure from which ITMP are referenced and coordinated. It was developed based on an implemented Process Lifecycle Model which was formalized according to CMPSI, ISO 9001:2000 Quality Management System Procedures, Procurement Systems, and Software Integration Requirements. The respective technical and support processes were then improved with using of ARIS, eEPC (ARIS, extended Event-driven Process Chain) Model, in order to provide a core/complete CPM model.

The ITMP obtain the guiding process structure, related to interoperability of CAD, ERP and Scheduling Systems which are used for CPM purposes. Using a process-centric approach (based on the ARIS-eEPC), the related services and data resources for each task were identified.

Referencing IFC Model data is provided via formally defined IFC views in the context of the respective tasks. This was achieved with the help of a formal specification using the Generalized Subset Definition Schema (GMSD) (Weise et al. 2003) developed at the TU-Dresden, rules for dynamic run-time filtering, and a dedicated service performing the actual view extraction for the specifically referenced CAD, ERP and Scheduling Systems. IFC schema objects were used as much as possible, with some needed extensions for CPM purposes. However, as the objective is to propose an integrated framework and show how IFC fits into it rather than develop a specific IFC extension model for CPM, this has been done only for selected examples.

Based on the envisaged configurations, an operational framework for CPM will be developed as an integrated client-server environment, enabling to plug in all component tools.

\section{INTEGRATED CPM MODEL}

Development of an integrated CPM Model requires a holistic approach, taking into consideration management items, software applications, product data descriptions and a web-based system infrastructure. 


\subsection{Construction Management Phases for Software Interoperability}

In order to formalize an integration methodology, encompassing the product and process information exchange within the CAD, ERP and Scheduling Systems which supports IFCs, the phase formalization principles: (1) General Project View, (2) Process Consistency, (3) Phase and Process Reviews etc. were developed. This approach provides the basis for the envisaged structure.

Furthermore, the Construction Management Phases for Software Interoperability which composed of five basic phases as: (1) Design, (2) Bidding Preparation, (3) Planning \& Construction, (4) Realization, and (5) Evaluation of Outcome and Feedback was improved with using of IDEF0 modeling methodology. In all phases specific databases and algorithms were used to provide suitable data structures which keep the information about function and content. These obtain re-use of requested information whenever needed. Bidding Preparation Phase of CMPSI was chosen to narrow the scope and to formalize a precise structure in this context.

\subsection{ISO9001:2000 Quality Management System CPM Procedures}

To establish a concurrent control system in terms of monitoring ongoing activities, there is a need for a generic procedural model. This should include assessment of current work activities which relies on performance standards, rules and regulations for guiding employee tasks and behaviors.

In order to support required aspects and to obtain a generic procedural model, ISO9001:2000 Quality Management System (ISO-QMS) was examined in detail for CPM purposes. To support a conceptual framework, the envisaged CPM structure was basically modeled according to interconnected procedures referencing ISO requirements. Moreover, four main procedures as; (1) General System, (2) Human Resource and Administrative, (3) Customer Relations, and (4) Project Management procedures were formalized. Based on these, the sub-procedures were developed to constitute supporting processes subsequently.

\subsection{Integrated CPM Processes}

Based on the implemented acquiescence in this research, two inter-related process formalizations as (1) Organizational Management Process (OMP) and (2) IT Management Processes (ITMP) were structured in ARIS-eEPC model. The main aim to use ARIS is to clearly recognize the common functions, events, resources and objects that are used both in OMP and ITMDP. This helps greatly to subsequently formalize the mappings between the different but interrelated data and functional definitions. According to the defined process activities, resources are primarily defined as IFC objects or, where necessary, as suggested new entities within an IFC model extension for CPM.

\subsubsection{Process Life Cycle Model for OMP}

To complete identified aspects, to develop integrated CPM process patterns and to define a process formalization structure, a Process Life Cycle Model was implemented for OMP formalization purposes. CMPSI, ISO, Procurement Systems and 
software integration requirements were brought together in this structure, thereby exposing an integrated model which meets the envisaged interoperability.

\subsubsection{Organizational Management Process}

The Organizational Management Process composed of interconnected processes based on a developed Process Life Cycle Model, was constructed to control whole process sequence.

According to Process Life Cycle Model, (1) initial analysis of bidding preparation phase of CMPSI, related to ISO-QMS procedures, organizational structures, procurement systems, and required services were possessed. This phase is fallowed by (2) a process design phase, during which the overall process structure is engineered, the resulting process model is designed, the resources examined and the mapping methodology is decided. This includes the modeling of organizational structures and services integrations. In the third phase (3) the designed processes were implemented with using of ARIS-eEPC. The main process was defined according to ISO Quality Management System's bidding preparation process which is identified under customer relations main procedure. The supporting processes (six interrelated process) such as job development, design coordination processes etc. under project management main procedure were also defined and used within bidding preparation structure. With bringing together of procurement systems and integration requirements for CAD, ERP and Scheduling Systems, OMP was obtained. After implementation of work flow (4) established processes were checked whether that they are supporting generic integration comprising seamless information flow by using IT systems. The formalized resources consistencies were controlled and the mapping structure was scrutinized in this regard.

\subsubsection{IT Management Processes}

IT Management Processes (ITMP) are defined in accordance with the CAD-ERPScheduling Systems interoperability needs and derived based on OMP. This includes the application sequence of the involved IT tools, their relations to processes, performing actors, input output and control information, and their general systemic interrelations in the IT environment. To show different level of system integration, Bidding Preparation Phase is organized in three subsequent structures as; IT Management (1) Design Process, (2) BOQ Process and (3) Scheduling Process.

\subsubsection{Process Mappings}

In order to provide a generic concept which identifies workflow participants, in terms of resources that can be addressed by CPM processes, we identified a mapping structure between CMPSI, OMP and ITMP. This helps us to examine IFC views which are used to implement IFC based management approach. The mappings between three structures provide 1-1 mapping (pairing) formalization. The CMPSI phase processes are used as main processes which are referenced by OMP processes as sub-processes. Also CMPSI resources are referenced by OMP resources as subresources. The same approach is used within OMP and ITMP mappings. 


\section{IFC DATA FORMALIZATION}

The IFC Object Model (IAI 2005) is essentially a project data model addressing the major data exchange requirements in the highly fragmented construction industry. It encompasses a large set of object definitions so that applications should or can implement only subsets of the full model. Such subsets are called IFC Views or, more generally, Data Exchange Use Cases. For practical use various such subsets are currently being defined applying more or less formal approaches (cf. ProIT 2004).

In this context, to enable interoperable use of IFC data in the General CPM Model and for the CAD-ERP and Scheduling applications the following procedure was applied: (1) The IT Management Processes defined in ARIS were examined with regard to IFC Data Exchange Use Cases that can or should be related to them. An example for such a use case is the data exchange from Architecture to Quantity Take-Off. (2) For each identified use case the relevant IFC objects and their relevant relationships are determined. They are then associated to the relevant organizational entities, and the relevant resource entities in the eEPC model. In the first case these will always be instances of IFC object classes, but in the second case they can be individual objects, property sets or whole model subsets. (3) Whenever model subsets need to be applied, the General Model Subset Definition Schema (GMSD) developed at the TU Dresden was used for the formal specification of the subset content on class level (Weise et al. 2003). (4) Runtime use of the IFC data was then provided via a specialized GMSD client which enables proper extraction of the specifically needed IFC instances in each particular situation. This was done interactively, whereas in the CPM model we provide only some requirements and hints to the user. Figure 1 below illustrates the principal ideas.

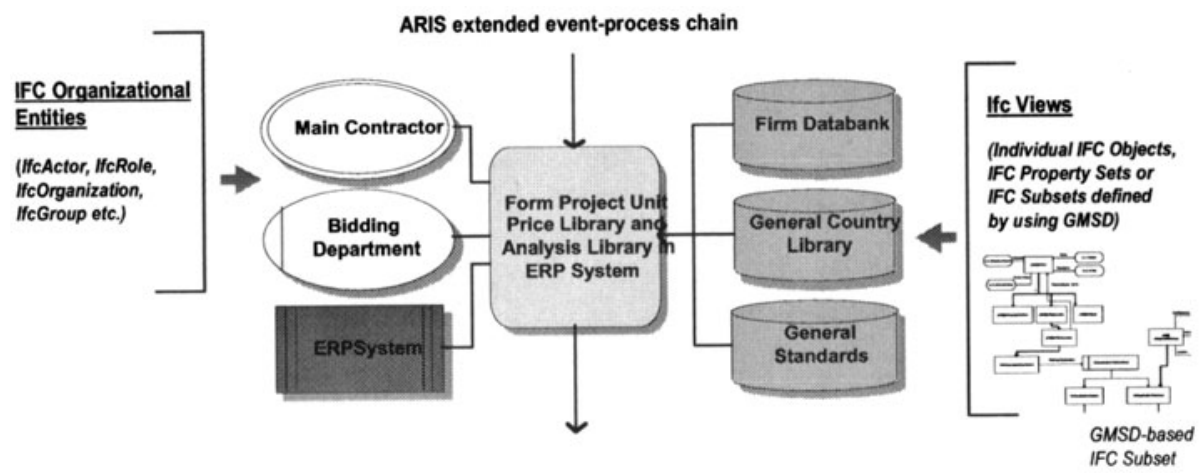

Figure 1. Schematic presentation of the association of IFC data to the CPM Model

\section{OPERATIONAL FRAMEWORK}

From the operational point of view, interoperability means the ability of the system components to work together in a coherent way for the solution of complex tasks. In this sense, the operational framework has to be structured and established according to a coherent process and information exchange paradigm as shown in Figure 2 below. It is comprised of 4 clearly defined layers: (1) Application Layer, (2) TSD Layer, (3) Management Process Layer, and (4) WPA Layer. 


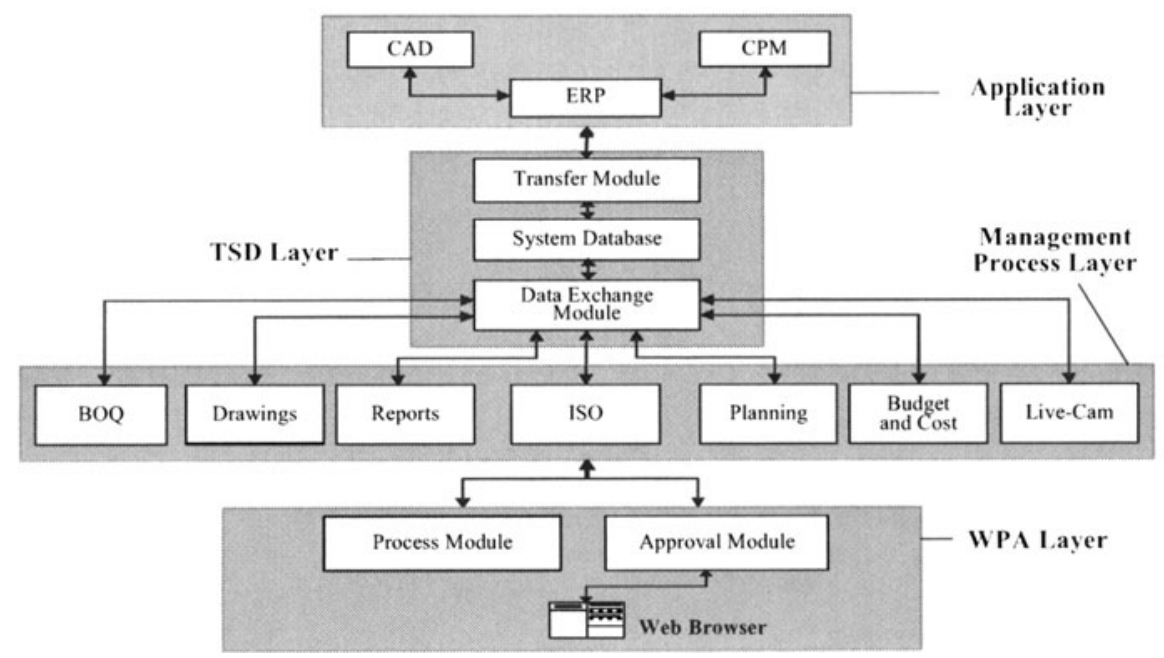

Figure 2. Suggested integrated Web-based CPM solution

\subsection{Application Layer}

The purpose of this layer is to support different types of value-add project activities, performed with the help of CAD, ERP and Scheduling applications. Typically, such applications are locally installed and used. They may provide some in-built interoperability but the basic input/output to/from them is achieved via the TSD Layer which is their gateway to the Web.

\section{TSD Layer}

This layer consists of a Transfer Module, a System Database and a Data Exchange Module. The information obtained from the Application Layer is stored in the System Database. This information covers the identified outcomes of the CAD, ERP and CPM applications. The Transfer Module supports the data exchange between the Application Layer and the System Database. Assuming that IFC data can be exported by the involved applications, this can be done with the help of a general purpose API in some convenient standard format (e.g. using ISO 10303-21 files and/or ifcXML). The stored database information is then processed by the Data Exchange Module which is the coordination module for the below layers, ensuring synchronous and asynchronous information flows in a coherent, standardized way.

\subsection{Management Process Layer}

The Management Process Layer consists of 7 different modules Drawing Module, Reports Module etc. that can perform and be managed separately and further process the data obtained from the TSD layer. Additionally, a Live-Cam Module can be provided to track the execution on the jobsite, and an ISO Module can be included for process support in accordance to ISO Quality Management procedures. 


\subsection{WPA Layer}

The WPA Layer provides facilities for (1) execution of the management processes and the related applications via the Internet, and (2) presentation of the obtained results to all stakeholders via a common Web Browser. The process workflows can be carried out using a standard schema and on every step the information can be checked and approved by the responsible persons who are attained by the virtual project organization.

\section{CONCLUSION}

In the above pages we outlined an integrated CPM model based on a logical conceptual schema starting with the specification of management along a number of welldefined steps towards the creation of an operational framework.

The major goals of the suggested approach is to denote the broad set of activities supported by multi-module application software that helps to manage the different work items and processes of a construction project. To reach these goals we have brought together state-of-the-art CAD-ERP-CPM technical interoperability concepts, a novel formalization and integration approach for ISO9001 quality management procedures, advanced IFC-based integration issues. Some clear benefits of the integral treatment of all CPM aspects on the basis of ARIS, ISO9001 and IFC were identified, especially with regard to IFC penetration in practice. Currently IFC use is still modest, mostly for CAD-based data exchange. With the developed CPM model a contribution towards its much broader use in ERP and CPM applications to support collaborative project management in all life cycle phases of the virtual enterprise of a construction project can be accomplished.

After completion of the system requirements, the suggested framework will be prototyped in the name of 'SanTez' (Industry Thesis) Project which is supported by Turkish Ministry of Industry. Subsequently, the prototype will be used within a construction site, in order to revise the necessary developments.

\section{REFERENCES}

1. IAI (2005): IFC/ifcXML Specifications. (C) International Alliance for Interoperability(http:/www. iai-international.org/Model/IFC(ifcXML)Specs.html )

2. ISO9001:2000 Quality Management System Requirements. Geneva, Switzerland.

3. Liebich T., Adachi Y., Forester J., Hyvarinen J., Karstila K \& Wix J. (2006): Industry Foundation Classes IFC2 3 . C International Alliance for Interoperability.

4. Luiten G., Froese T., Björk B-C., Cooper G., Junge R., Karstila K. \& Oxman R. (1993): An information Reference Model for Achitecture, Engineering and Construction. In: Mathur K. D., Betts M. P. \& Tham K.W. (eds.) The Management of Informations Technology for Construction, World Scientific Publishing, Singapore.

5. ProIT (2004): ProIT: Product Model Data in the Construction Process. (C) IAI International Solutions.

6. Scheer A.-W. (1996): ARIS - House of Business Engineering. Research Report, Heft 133, IWi an der Unversităt des Saarlands, Saarbrücken, Germany.

7. Weise M., Katranuschkov P. \& Scherer R. J. (2003): Generalised Model Subset Definition Schema. In: Amor R. (ed.) "Construction IT: Bridging the Distance", Proc. of the CIB-W78 Workshop, 2325 April 2003, Waiheke Island, Auckland, New Zcaland. 